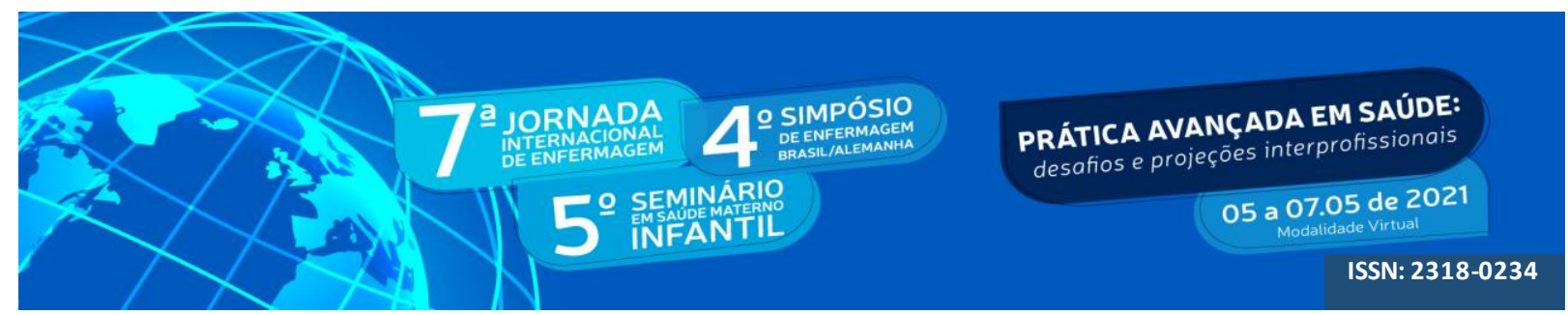

DOI: http://doi.org/10.48195/jie2021-045

\title{
MEDICINAS TRADICIONAIS, COMPLEMENTARES E INTEGRATIVAS NA HIPERTENSÃO ARTERIAL SISTÊMICA: REVISÃO INTEGRATIVA ${ }^{1}$
}

\section{Daiana Cristina Wickert ${ }^{2}$; Maria Denise Schimith ${ }^{3}$; Laís Mara Caetano da Silva ${ }^{4}$; Luiza Camila Jerke $^{5}$; Dedabrio Marques Gama ${ }^{6}$; Marcelo Nunes da Silva Femandes ${ }^{7}$}

\begin{abstract}
RESUMO
Objetivo: identificar as evidências científicas acerca da utilização das medicinas tradicionais, complementares e integrativas para o controle da hipertensão arterial sistêmica em adultos. Método: revisão integrativa realizada na PUBMED, em maio de 2019. Resultados: foram selecionados 32 estudos, todos disponíveis em inglês, dentre estes, seis (18,8\%) publicados nos Estados Unidos da América, 24 (75\%) realizados com pacientes/usuários hipertensos, e os cenários que mais apareceram foram a Atenção Primária à Saúde, hospitais e clínicas, com seis $(18,8 \%)$ estudos cada. Predominaram ensaios clínicos randomizados, oito $(25 \%)$, e pesquisas com produtos naturais/fitoterápicos ou plantas medicinais, aparecendo em 21 (65,7\%) estudos, sendo que as cápsulas de Ginseng Americano 3g e o consumo de $150 \mathrm{ml} /$ dia de suco de Romã mostraram evidências significativas. Conclusões: o estudo mostrou uma prevalência de utilização de produtos naturais/fitoterápicos ou plantas medicinais no controle da hipertensão arterial sistêmica, com evidências significativas para o o Ginseng Americano e suco de Romã.
\end{abstract}

Palavras-chave: Hipertensão; Medicina Integrativa; Medicina Tradicional; Terapias complementares.

\begin{abstract}
Objective: to identify scientific evidence about the use of traditional, complementary and integrative medicines for the control of systemic arterial hypertension in adults. Method: an integrative review carried out at PUBMED, in May 2019. Results: 32 studies were selected, all available in English, among them, six $(18.8 \%)$ published in the United States of America, $24(75 \%)$ carried out with patients / hypertensive users, and the scenarios that most appeared were Primary Health Care, hospitals and clinics, with six $(18.8 \%)$ studies each. Randomized clinical trials predominated, eight $(25 \%)$, and research with natural / herbal products or medicinal plants, appearing in $21(65.7 \%)$ studies, with American Ginseng capsules 3g and the consumption of $150 \mathrm{ml} /$ day of juice of Pomegranate showed significant evidence. Conclusions: the study showed a prevalence of the use of natural / herbal products or medicinal plants in the control of systemic arterial hypertension, with significant evidence for American Ginseng and Pomegranate juice.
\end{abstract}

Key Words: Hypertension; Integrative Medicine; Medicine Traditional; Complementary Therapies.

\footnotetext{
${ }^{1}$ Revisão Integrativa.

${ }^{2}$ Enfermeira. Mestranda em Enfermagem- PPGEnf/UFSM. daianacristinaw@gmail.com

${ }^{3}$ Enfermeira. Orientadora. Profess ora do Curso de Enfermagem - UFSM. ma.denise2011@gmail.com

${ }^{4}$ Enfermeira. Profess ora do Curso de Enfermagem - UFSM. lais.silva@ufsm.br

${ }^{5}$ Graduanda em Enfermagem- UFSM. luizajerke@gmail.com

${ }^{6}$ Enfermeiro. Mestrando em Enfermagem- PPGEnf/UFSM. dedabrio.gama@gmail.com

${ }^{7}$ Enfermeiro. Doutorando em Enfermagem - PPGEnf/UFRGS. marcelonsf@gmail.com
} 


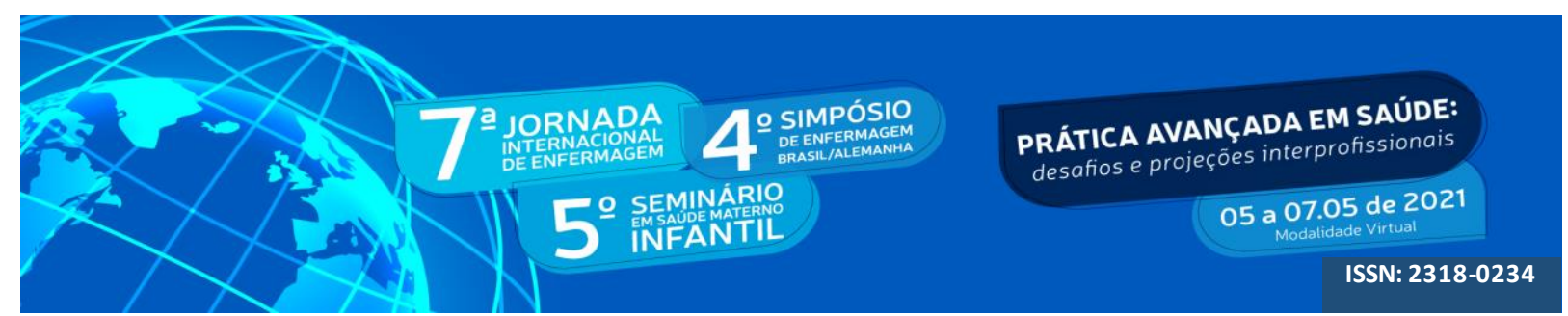

\section{INTRODUÇÃO}

Por ser uma doença silenciosa nos estágios iniciais, o diagnóstico da hipertensão arterial sistêmica (HAS), por vezes, acaba sendo tardio, e o tratamento dificultado devido ao acesso ao mesmo. Percebe-se que os ganhos são significativos com o diagnóstico precoce e o tratamento adequado, visto que as complicações da HAS geram altos custos (WHO, 2013a).

Para isso, tem-se trabalhado com as questões da prevenção primária e controle da HAS mundialmente. No entanto, sabe-se que os mesmos são complexos e precisam ser planejados de uma forma acessível e sustentável para todos os países, visto que envolve governos, sociedade, a indústria de alimentos, bebidas e tabaco (WHO, 2013a). Dentre as estratégias para prevenção e controle da HAS, pode-se pensar nas medicinas tradicionais, complementares e integrativas (MTCI), visto que, mesmo com o uso de medicamentos, um terço das pessoas com HAS acaba morrendo por acidente vascular encefálico (AVE), infarto agudo do miocárdio (IAM) ou insuficiência cardíaca (VICTOR, 2012).

Assim cabe a reflexão de que a terapia medicamentosa é importante, mas que apenas ela pode não ser suficiente para controlar ou mesmo reverter a HAS. Frente a isso, elaborouse a seguinte pergunta de revisão: quais as evidências científicas acerca da utilização das medicinas tradicionais, complementares e integrativas para o controle da hipertensão arterial sistêmica em adultos?

\section{OBJETIVO}

Identificar as evidências científicas acerca da utilização das medicinas tradicionais, complementares e integrativas para o controle da hipertensão arterial sistêmica em adultos.

\section{METODOLOGIA}

Trata-se de um estudo de revisão integrativa (RI) da literatura, sendo que a mesma permite conhecer o que existe publicado sobre um tema e assim, identificar possíveis lacunas do conhecimento ou ainda, auxiliar na prática baseada em evidência para aplicação clínica no 


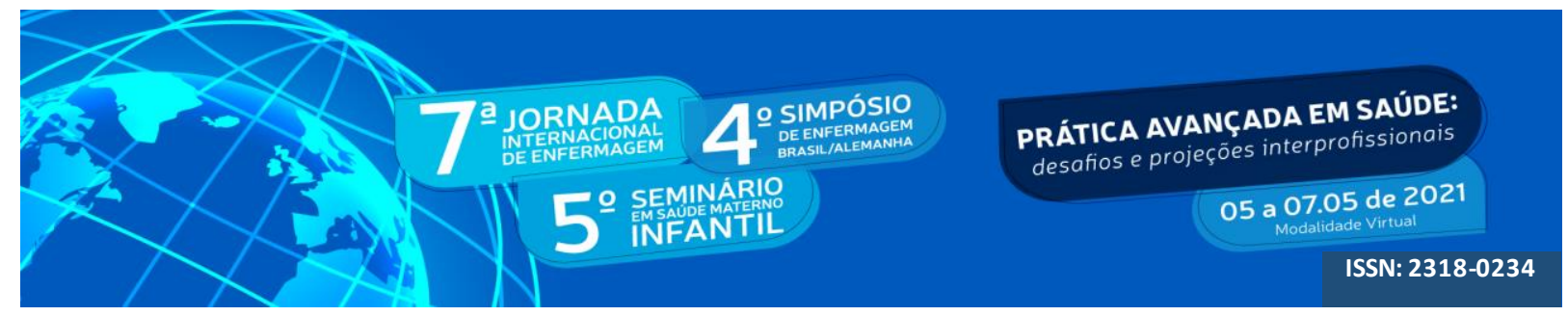

cuidado em saúde (MENDES; SILVEIRA; GALVÃO, 2008).

Para desenvolver o presente estudo, foram percorridas seis etapas: identificação do tema e elaboração da questão de pesquisa, estabelecimento de critérios de inclusão e exclusão, seleção de base de dados e dos estudos, definição das informações a serem extraídas dos estudos selecionados, avaliação das evidências e análise, e apresentação da síntese do conhecimento produzido (MENDES; SILVEIRA; GALVÃO, 2008).

Contou-se com o auxílio de uma bibliotecária da Biblioteca Central (BC) da Universidade Federal de Santa Maria (UFSM), para definir a biblioteca e a estratégia de busca para amostragem de artigos a serem avaliados. Assim, optou-se pela biblioteca US National Library of Medicine (PubMed), sendo o acesso realizado via Comunidade Acadêmica Federada (CAFe), no portal de periódicos da Coordenação de Aperfeiçoamento de Pessoal de Nível Superior. A escolha da biblioteca PubMed se deu devido a temática das MTCI ser mundialmente pesquisada e não se restringir apenas ao Brasil e a Enfermagem.

As buscas foram realizadas no mês de maio de 2019, sendo utilizado o Mesh term complementary therapies, e as palavras: integrative and complementary practices; alternative medicine; hypertension. Assim, a estratégia de busca adotada foi: "complementary therapies" OR "integrative and complementary practices" OR "alternative medicine" AND "hypertension". Não se utilizou recorte temporal, sendo os critérios de inclusão: pesquisa com participantes com 18 anos ou mais, artigos em inglês, português ou espanhol e artigos disponíveis ou disponibilizados pelos autores. Foram excluídos os artigos que não responderam a pergunta de revisão. Para os artigos não disponíveis na íntegra, foram realizados contatos com os autores e buscas para acessar aos mesmos, entretanto, ainda restaram quatro artigos indisponíveis.

\section{RESULTADOS E DISCUSSÃO}

Foram selecionados 32 artigos e extraídos dados referentes a: identificação do artigo, nível de evidência, referência, objetivo, delineamento e população, principais resultados, ano de publicação e país de realização do estudo. Todos os aspectos éticos foram respeitados, sendo os autores referenciados adequadamente e os resultados das pesquisas apresentados de 


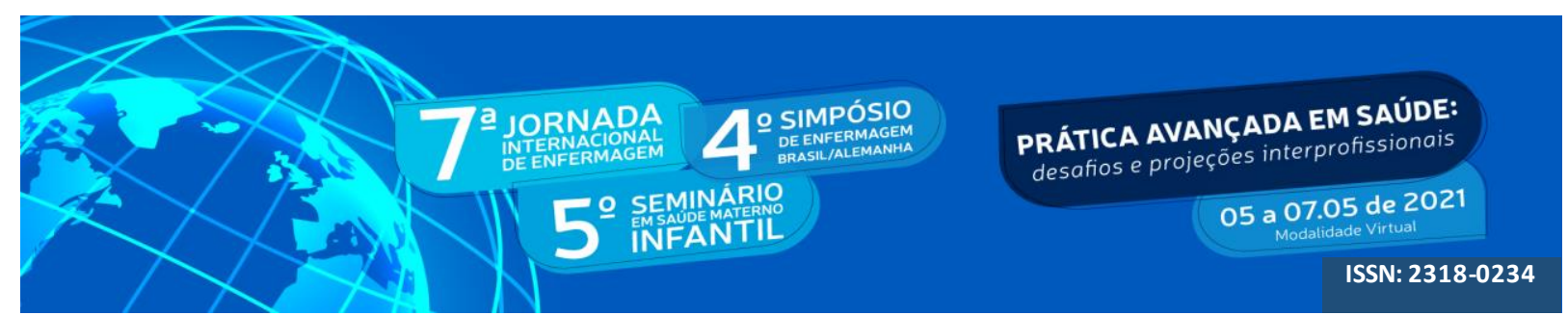

forma fidedigna. O processo de seleção dos estudos está ilustrado na Figura 1.

Figura 1: Fluxo do processo de seleção dos estudos acerca da utilização das medicinas tradicionais, complementares e integrativas para controle da hipertensão.

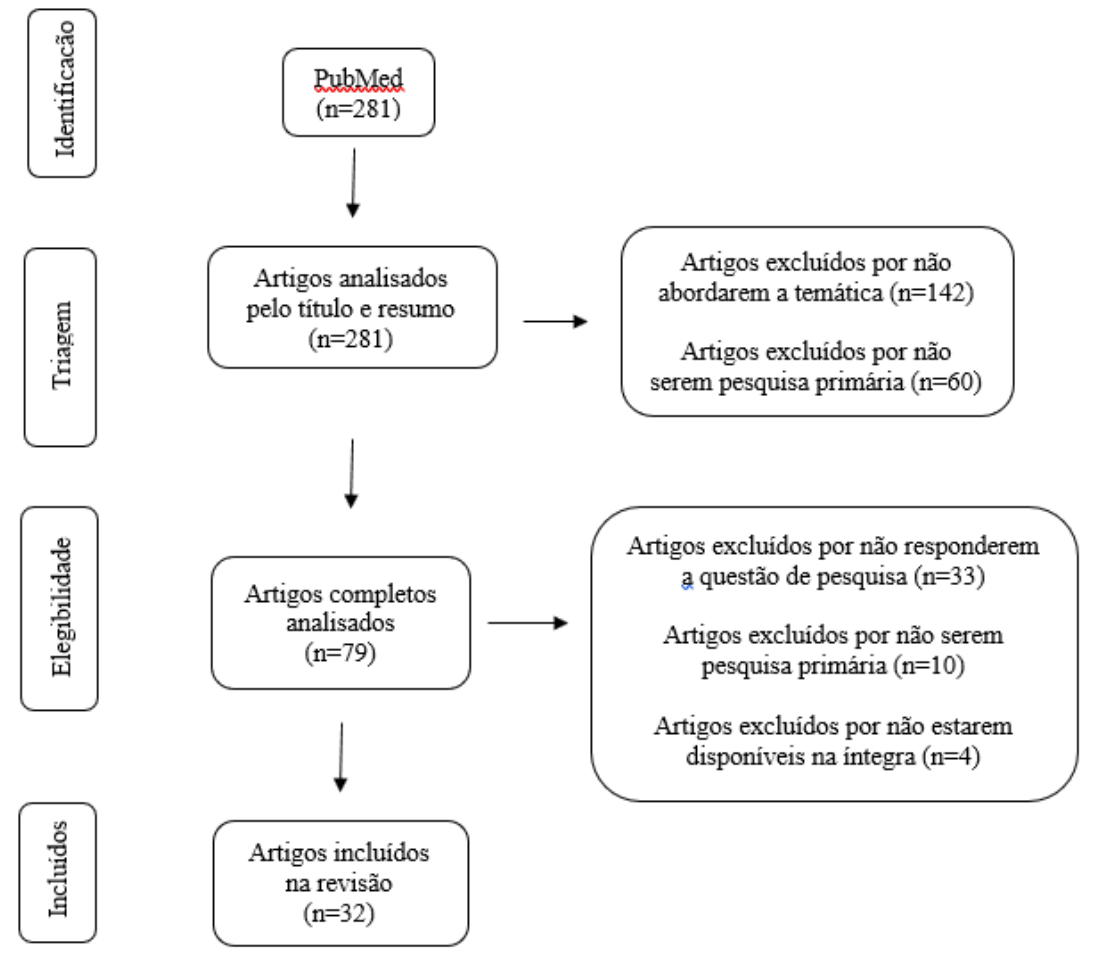

Fonte: Figura elaborada pelos autores com base no diagrama PRISMA (MOHER et al., 2009).

A fim de facilitar a compreensão dos estudos e manter a fidedignidade dos resultados, os artigos estão representados pela identificação A (artigos) seguido por numeração ordinal $(1,2,3 \ldots 32)$, conforme Quadro 1.

Quadro 1: Identificação e referências dos estudos selecionados para a revisão.

\begin{tabular}{|l|l|}
\hline ID & Refe rência \\
\hline A1 & $\begin{array}{l}\text { AL-TABAKHA, M. et al. Evaluation of bloodletting cupping therapy in the management of } \\
\text { hypertension. Journal Of Pharmacy And Bioallied Sciences, [s.l.], v. 10, n. 1, p.1-9, } 2018 .\end{array}$ \\
\hline A2 & $\begin{array}{l}\text { ABDULLAH, N. et al. Utilization of Complementary and Alternative Medicine in Multiethnic } \\
\text { Population: The Malaysian Cohort Study. Journal Of Evidence-based Integrative Medicine, } \\
\text { [s.l.], v. 23, p.1-19, jan. 2018. }\end{array}$ \\
\hline A3 & $\begin{array}{l}\text { WOLFF, M. et al. Yoga - a laborious way to well-being: patients' experiences of yoga as a } \\
\text { treatment for hypertension in primary care. Scandinavian Journal Of Primary Health Care, } \\
\text { [s.l.], v. 35, n. 4, p.360-368, 2 out. 2017. }\end{array}$ \\
\hline
\end{tabular}




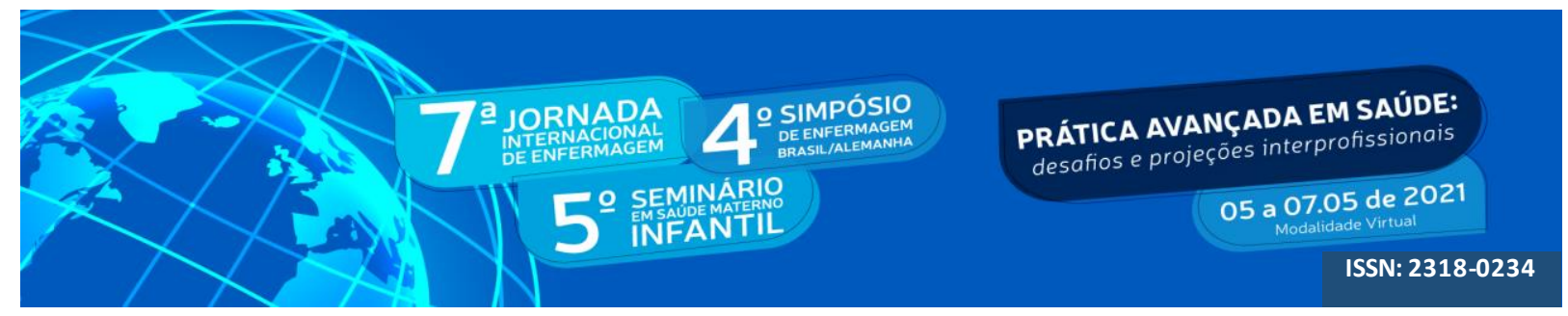

\begin{tabular}{|c|c|}
\hline$\overline{\mathrm{A} 4}$ & $\begin{array}{l}\text { LIWA, A. et al. Herbal and Alternative Medicine Use in Tanzanian Adults Admitted with } \\
\text { Hypertension-Related Diseases: A Mixed-Methods Study. International Journal Of } \\
\text { Hypertension, [s.1.], v. 2017, p.1-9, } 2017 \text {. }\end{array}$ \\
\hline A5 & $\begin{array}{l}\text { LULEBO, A. M. et al. Prevalence and determinants of use of complementary and alternative } \\
\text { medicine by hypertensive patients attending primary health care facilities in Kinshasa, } \\
\text { Democratic Republic of the Congo: a cross-sectional study. Bmc Complementary And } \\
\text { Alte rnative Medicine, [s.l.], v. 17, n. 1, p.1-9, } 8 \text { abr. } 2017 \text {. }\end{array}$ \\
\hline A6 & $\begin{array}{l}\text { GOERTZ, C, M. et al. Effect of Spinal Manipulation of Upper Cervical Vertebrae on Blood } \\
\text { Pressure: Results of a Pilot Sham-Controlled Trial. Journal Of Manipulative And } \\
\text { Physiological Therape utics, [s.1.], v. 39, n. 5, p.369-380, jun. } 2016 \text {. }\end{array}$ \\
\hline A7 & $\begin{array}{l}\text { ESAKKIMUTHU, S. et al. Quantitative ethnomedicinal survey of medicinal plants given for } \\
\text { cardiometabolic diseases by the non-institutionally trained siddha practitioners of Tiruvallur } \\
\text { district, Tamil Nadu, India. Journal Of Ethnopharmacology, [s.1.], v. 186, p.329-342, jun. } \\
2016 \text {. }\end{array}$ \\
\hline A8 & $\begin{array}{l}\text { ALEYEIDI, N. A. et al. Effects of wet-cupping on blood pressure in hypertensive patients: a } \\
\text { randomized controlled trial. Journal Of Integrative Medicine, [s.1.], v. 13, n. 6, p.391-399, } \\
\text { nov. 2015. }\end{array}$ \\
\hline A9 & $\begin{array}{l}\text { TULUNAY, M. et al. Herbal medicine use among Turkish patients with chronic } \\
\text { diseases. Journal Of Inte rcultural Ethnopharmacology, [s.1.], v. 4, n. 3, p.217-220, } 2015 .\end{array}$ \\
\hline $\bar{A} 10$ & $\begin{array}{l}\text { SIBBRITT, D. et al. Use of Complementary and Alternative Medicine in Women With Heart } \\
\text { Disease, Hypertension and Diabetes (from the Australian Longitudinal Study on Women's } \\
\text { Health). The American Journal Of Cardiology, [s.l.], v. 115, n. 12, p.1691-1695, jun. } 2015 .\end{array}$ \\
\hline A11 & $\begin{array}{l}\text { MERATI, G. et al. Cardiovascular adaptation to mudpack theral } \\
\text { treated with different antihypertensive drugs. European Reviev } \\
\text { Pharmacological Sciences, Pádua, v. 18, p.2544-2550, } 2014 .\end{array}$ \\
\hline A12 & $\begin{array}{l}\text { TSAI, D. et al. Prescription pattern of Chinese herbal products for hypertension in Taiwan: A } \\
\text { population-based study. Journal Of Ethnopharmacology, [s.1.], v. 155, n. 3, p.1534-1540, set. } \\
\text { 2014. }\end{array}$ \\
\hline A13 & $\begin{array}{l}\text { LEE, K. et al. Hypertensive patients' preferences for complementary and alternative medicine } \\
\text { and the influence of these preferences on the adherence to prescribed } \\
\text { medication. Complementary The rapies In Clinical Practice, [s.1.], v. 20, n. 2, p.99-105, maio } \\
2014 \text {. }\end{array}$ \\
\hline A14 & $\begin{array}{l}\text { A KRETCHY, I.; OWUSU-DAAKU, F.; DANQUAH, S.. Patterns and determinants of the use } \\
\text { of complementary and alternative medicine: a cross-sectional study of hypertensive patients in } \\
\text { Ghana. B mc Complementary And Alte rnative Medicine, [s.l.], v. 14, n. 1, p.1-7, } 4 \text { fev. } 2014 .\end{array}$ \\
\hline A15 & $\begin{array}{l}\text { ALI-SHTAYEH, M. S. et al. Complementary and alternative medicine (CAM) use among } \\
\text { hypertensive patients in Palestine. Complementary The rapies In Clinical Practice, [s.1.], v. } \\
\text { 19, n. 4, p.256-263, nov. } 2013 \text {. }\end{array}$ \\
\hline A16 & $\begin{array}{l}\text { MOLLAOğLU, M.; ACIYURT, A. Use of complementary and alternative medicine among } \\
\text { patients with chronic diseases. Acta Clinica Croatica, Sivas, v. 52, p.181-188, } 2013 .\end{array}$ \\
\hline A17 & $\begin{array}{l}\text { SHUKLA, G. et al. Efficacy of Virechana and Basti Karma with Shamana therapy in the } \\
\text { management of essential hypertension: A comparative study. Ayu (an International Quarterly } \\
\text { Journal Of Research In Ayurveda), [s.1.], v. 34, n. 1, p.70-76, 2013. Medknow. }\end{array}$ \\
\hline A18 & $\begin{array}{l}\text { MUCALO, Iva et al. Effect of American ginseng (Panax quinquefolius L.) on arterial stiffness } \\
\text { in subjects with type-2 diabetes and concomitant hypertension. Journal Of } \\
\text { Ethnopharmacology, [s.1.], v. 150, n. 1, p.148-153, out. } 2013 \text {. }\end{array}$ \\
\hline
\end{tabular}




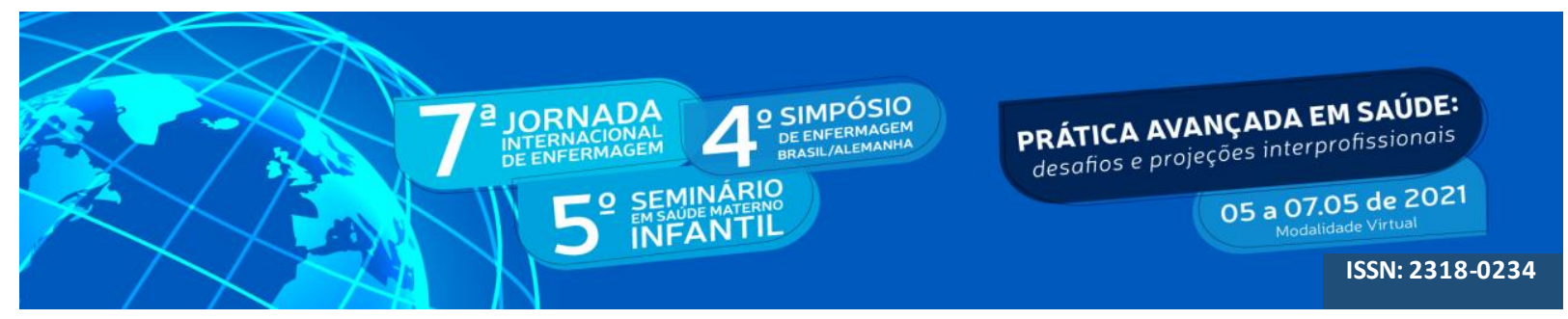

\begin{tabular}{|c|c|}
\hline$\overline{\text { A19 }}$ & $\begin{array}{l}\text { WOO, K. S. et al. Cardiovascular Protective Effects of Adjunctive Alternative Medicine (Salvia } \\
\text { miltiorrhizaandPueraria lobata) in High-Risk Hypertension. Evidence-based Complementary } \\
\text { And Alternative Medicine, [s.l.], v. 2013, p.1-8, } 2013 \text {. }\end{array}$ \\
\hline$\overline{\mathrm{A} 20}$ & $\begin{array}{l}\text { ASGARY, S. et al. Clinical Evaluation of Blood Pressure Lowering, Endothelial Function } \\
\text { Improving, Hypolipidemic and Anti-Inflammatory Effects of Pomegranate Juice in } \\
\text { Hypertensive Subjects. Phytothe rapy Research, [s.l.],v. 28, n. 2, p.193-199, } 21 \text { mar. } 2013 .\end{array}$ \\
\hline A21 & $\begin{array}{l}\text { KOC, Z.; TOPATAN, S.; SAGLAM, Z. Use of and attitudes toward complementary and } \\
\text { alternative medicine among midwives in Turkey. European Journal Of Obstetrics \& } \\
\text { Gyne cology And Reproductive Biology, [s.1.], v. 160, n. 2, p.131-136, fev. } 2012 \text {. }\end{array}$ \\
\hline A22 & $\begin{array}{l}\text { BRADLEY, R. et al. Observed Changes in Risk during Naturopathic Treatment of } \\
\text { Hypertension. Evidence-based Complementary And Alte rnative Medicine, [s.l.], v. 2011, } \\
\text { p.1-7, } 2011 .\end{array}$ \\
\hline A23 & $\begin{array}{l}\text { OSAMOR, P.; OWUMI, B. Complementary and alternative medicine in the management of } \\
\text { hypertension in an urban Nigerian community. B mc Complementary And Alte rnative } \\
\text { Medicine, [s.l.], v. 10, n. 1, p.1-9, } 19 \text { jul. 2010. }\end{array}$ \\
\hline A24 & $\begin{array}{l}\text { TANG, H.; HARMS, V.; VEZEAU, T. An Audio Relaxation Tool for Blood Pressure } \\
\text { Reduction in Older Adults. Ge riatric Nursing, [s.1.], v. 29, n. 6, p.392-401, nov. } 2008 .\end{array}$ \\
\hline A25 & $\begin{array}{l}\text { TOPRAK, D.; DEMIR, S. Treatment Choices of Hypertensive Patients in Turkey. Behavioral } \\
\text { Medicine, [s.1.], v. 33, n. 1, p.5-10, abr. 2007. }\end{array}$ \\
\hline A26 & $\begin{array}{l}\text { BELL, R. A. et al. CAM Use Among Older Adults Age } 65 \text { or Older with Hypertension in the } \\
\text { United States: General Use and Disease Treatment. The Journal Of Alte rnative And } \\
\text { Complementary Medicine, [s.1.], v. 12, n. 9, p.903-909, nov. } 2006 \text {. }\end{array}$ \\
\hline A27 & $\begin{array}{l}\text { YEH, G. Y.; DAVIS, R. B.; PHILLIPS, R. S. Use of Complementary Therapies in Patients } \\
\text { With Cardiovascular Disease. The American Journal Of Cardiology, [s.1.], v. 98, n. 5, p.673- } \\
\text { 680, set. } 2006 .\end{array}$ \\
\hline A28 & $\begin{array}{l}\text { MAHFUDZ, A. S.; CHAN, S. C. Use of Complementary Medicine Amongst Hypertensive } \\
\text { Patients in a Public Primary Care Clinic in Ipoh. The Medical Journal Of Malaysia, Ipoh, v. } \\
\text { 60, p.454-459, 2005. }\end{array}$ \\
\hline A29 & $\begin{array}{l}\text { CONNELL, P.; MCKEVITT, C.; WOLFE, C. Strategies to manage hypertension: a qualitative } \\
\text { study with black Caribbean patients. British Journal Of Ge neral Practice, Caribe, p.357-361, } \\
2005 .\end{array}$ \\
\hline A30 & $\begin{array}{l}\text { OKE, D. A.; BANDELE, E. O. Misconceptions of Hypertension. Journal Of The National } \\
\text { Medical Association, Lagos, v. 96, p.1221-1224, } 2004 .\end{array}$ \\
\hline A31 & $\begin{array}{l}\text { LEE, M. et al. Effects of Qi-therapy on blood pressure, pain and psychological symptoms in the } \\
\text { elderly: a randomized controlled pilot trial. Complementary The rapies In Medicine, [s.1.], v. } \\
\text { 11, n. 3, p.159-164, set. } 2003 \text {. }\end{array}$ \\
\hline A32 & $\begin{array}{l}\text { GOERTZ, C. H. et al. Treatment of Hypertension with Alternative Therapies (THAT) Study: a } \\
\text { randomized clinical trial. Journal Of Hypertension, Minneapolis, v. 20, p.2063-2068, } 2002 .\end{array}$ \\
\hline
\end{tabular}

Fonte: Autores.

Todos os artigos estavam disponíveis no idioma inglês. Quanto ao país de publicação,

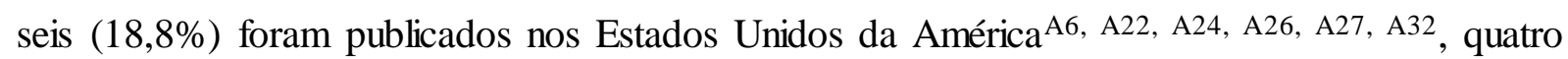
$(12,5 \%)$ na Turquia ${ }^{\mathrm{A} 9}, \mathrm{~A} 16, \mathrm{~A} 21, \mathrm{~A} 25$, Malásia com três $(9,5 \%)$ publicações ${ }^{\mathrm{A} 2}$, A13, A28, Índia ${ }^{\mathrm{A} 7, \mathrm{~A} 17} \mathrm{e}$ Nigéria ${ }^{\mathrm{A} 23}$, A300 com duas (6,3\%) publicações cada, Taiwan ${ }^{\mathrm{A} 12}$, Tanzânia ${ }^{\mathrm{A} 4}$, Suécia ${ }^{\mathrm{A} 3}$, República Democrática do Congo ${ }^{\mathrm{A} 5}$, Palestina ${ }^{\mathrm{A} 15}$, Itália ${ }^{\mathrm{A} 11}$, Irã ${ }^{\mathrm{A} 20}$, Hong Kong $^{\mathrm{A} 19}$, Gana ${ }^{\mathrm{A} 14}$, Emirados Árabes Unidos ${ }^{\mathrm{A} 1}$, Correia do $\mathrm{Sul}^{\mathrm{A} 31}$, 


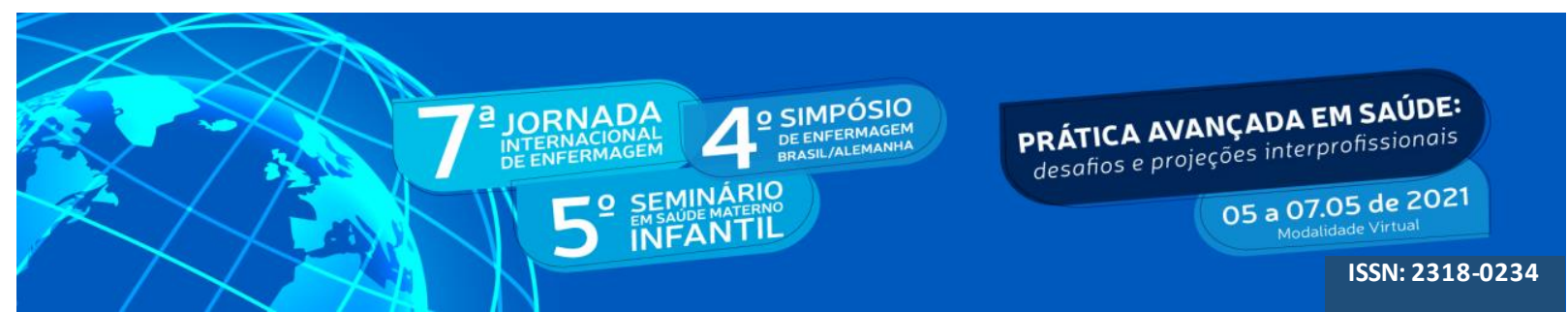

Caribe $^{\mathrm{A} 29}$, CanadáA18, Austrália ${ }^{\mathrm{A} 10}$ e Arábia Saudita ${ }^{\mathrm{A} 8}$ com uma $(3,1 \%)$ publicação cada. A maioria dos estudos foi publicado em 2013, com seis (18,8\%) publicações A15, A16, A17, A18, A19, A20, 2014 teve quatro (12,5\%) publicações ${ }^{\mathrm{A} 11, \mathrm{~A} 12, \mathrm{~A} 13, \mathrm{~A} 14}, 2017^{\mathrm{A} 3}, \mathrm{~A} 4, \mathrm{~A} 5$ e $2015^{\mathrm{A} 8, \mathrm{~A} 9, \mathrm{~A} 10}$ com três $(9,5 \%)$ publicações cada, 2018 ${ }^{\mathrm{A} 1}, \mathrm{~A} 2,2016^{\mathrm{A} 6}, \mathrm{~A} 7,2006^{\mathrm{A} 26, \mathrm{~A} 27}$ e $2005^{\mathrm{A} 28, \mathrm{~A} 29}$ com duas $(6,3 \%)$, e $2012^{\mathrm{A} 21}, 2011^{\mathrm{A} 22}, 2010^{\mathrm{A} 23}, 2008^{\mathrm{A} 24}, 2007^{\mathrm{A} 25}, 2004^{\mathrm{A} 30}, 2003^{\mathrm{A} 31}, 2002^{\mathrm{A} 32}$ com apenas uma $(3,1 \%)$ publicação cada.

No que se refere a população pesquisada, 24 (75\%) realizaram suas pesquisas diretamente com as pessoas com hipertensão A2, A3, A4, A5, A6, A8, A9, A12, A13, A14, A15, A16, A17, A18, A19, A20, A23, A25, A27. A28, A29, A30, A31, A32, dois (6,3\%) avaliaram prontuários ${ }^{\mathrm{A} 1, \mathrm{~A} 22}$, um $(3,1 \%)$ estudo foi realizado com médicos ${ }^{\mathrm{A} 7}$ e um $(3,1 \%)$ com parteiras ${ }^{\mathrm{A} 21}$. Ainda, um $(3,1 \%)$ estudo $^{\mathrm{A} 10}$ foi realizado somente com mulheres, já outros três $(9,5 \%)$ estudos realizados com idosos ${ }^{\mathrm{A} 11, \mathrm{~A} 24, \mathrm{~A} 26}$.

Quanto ao cenário de realização dos estudos, a Atenção Primária à Saúde A3, A5, A13, A21, A23, A28, hospitais ${ }^{\mathrm{A} 4}, \mathrm{~A} 8, \mathrm{~A} 14, \mathrm{~A} 16, \mathrm{~A} 17, \mathrm{~A} 19$ e clínicas $^{\mathrm{A} 6}, \mathrm{~A} 18, \mathrm{~A} 20, \mathrm{~A} 29, \mathrm{~A} 31, \mathrm{~A} 32$ tiveram seis $(18,8 \%)$ estudos cada, cinco $(15,7 \%)$ foram realizados em ambulatórios ${ }^{\mathrm{A} 9}$, A15, A22, A25, A30, e outros cinco $(15,7 \%)$ em bancos de dados de pesquisas populacionais ${ }^{\mathrm{A} 2}$, A10, A12, A26, A27. Ainda, os cenários de Serviço Personalizado de Atendimento $(S P A)^{\mathrm{A} 11}$, Centro de Hijama ${ }^{\mathrm{A} 1}$, Taluks (subdistritos de um município da Índia) ${ }^{\mathrm{A} 7}$ e Centro residencial ${ }^{\mathrm{A} 24}$ tiveram um $(3,1 \%)$ estudo cada.

No que se refere aos delineamentos de estudos, houve prevalência de Ensaio Clínico Randomizados (ECR) com oito estudos (25\%), como pode ser observado na Tabela 1. Ainda, quanto a categorização dos níveis de evidência dos estudos, a mesma foi realizada pela classificação proposta por Melnyk e Fineout-Overholt (2011), de acordo com as questões clínicas, conforme Tabela 1.

Tabela 1: Delineamentos e níveis de evidência dos estudos incluídos no corpus da revisão.

\begin{tabular}{c|c|c|c}
\hline Identificação do es tudo & Questão clínica & NE & Delineamento \\
\hline $\mathrm{A} 2, \mathrm{~A} 5, \mathrm{~A} 14, \mathrm{~A} 16, \mathrm{~A} 25$ & Prognóstico/Etiologia & 4 & Transversal analítico \\
\hline $\mathrm{A} 4, \mathrm{~A} 23$ & Prognóstico/Etiologia & 4 & Misto \\
\hline $\mathrm{A} 9, \mathrm{~A} 21, \mathrm{~A} 26, \mathrm{~A} 27, \mathrm{~A} 28 \mathrm{~A} 30$ & Prognóstico/Etiologia & 4 & Transversal descritivo \\
\hline $\mathrm{A} 10, \mathrm{~A} 12, \mathrm{~A} 15$ & Prognóstico/Etiologia & 2 & Coorte \\
\hline $\mathrm{A} 3, \mathrm{~A} 7, \mathrm{~A} 13, \mathrm{~A} 29$ & Significado/Experiência & 2 & Qualitativo \\
\hline
\end{tabular}




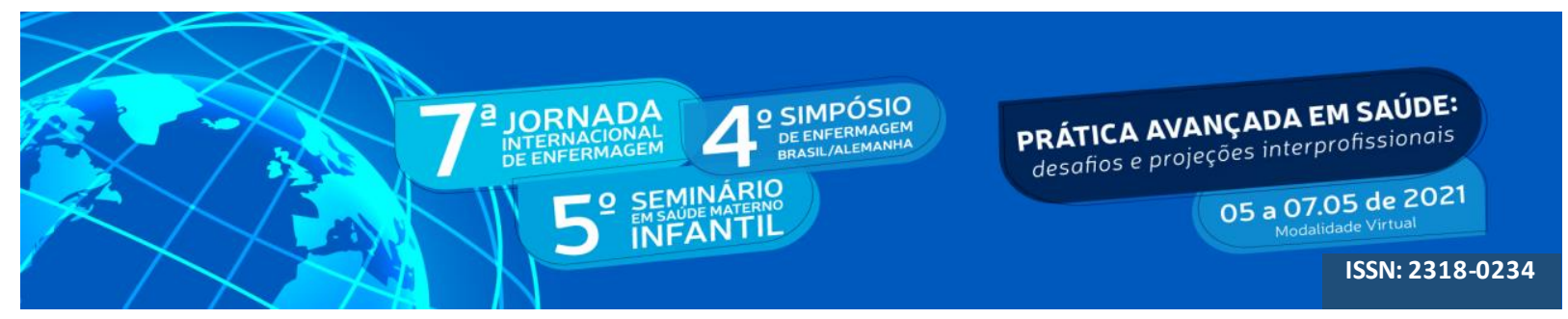

\begin{tabular}{c|c|c|c}
\hline A1 & Tratamento/Intervenção & 4 & Caso controle \\
\hline $\begin{array}{c}\text { A6, A8, A17, A18, A19, A20, A21, } \\
\text { A32 }\end{array}$ & Tratamento/Intervenção & 2 & ECR \\
\hline A11 & Tratamento/Intervenção & 3 & ECSR \\
\hline A22 & Tratamento/Intervenção & 4 & Coorte \\
\hline A24 & Tratamento/Intervenção & 6 & Estudo piloto descritivo \\
\hline
\end{tabular}

Fonte: Autores.

Legenda: NE= nível de evidência; ECR=Ensaio Clínico Randomizado; ECSR: Ensaio clínico sem randomização.

As pesquisas e discussões relacionadas as MTCI vem crescendo fortemente no cenário internacional (WHO, 2013b). Tendo como eixo central a promoção da saúde e prevenção de agravos, pode-se afirmar que as MTCI são importantes terapêuticas para a HAS. Nesse sentido, diversas pesquisas vem sendo desenvolvidas mundialmente, mostrando evidências da eficácia das MTCI no controle da HAS, como apresenta Wong et al. (2018), em sua revisão, no entanto, ainda existem lacunas importantes a serem exploradas.

Na presente RI, no que se refere a utilização das MTCI e os tipos que apareceram nos estudos, houve predomínio de produtos naturais/fitoterápicos ou plantas medicinais, aparecendo em $22(68,8 \%)$ estudos, conforme a Tabela 2.

Tabela 2: Prevalência das medicinas tradicionais, complementares e integrativas nos estudos.

\begin{tabular}{l|c}
\hline \multicolumn{1}{c|}{ Te rapia alte rnativa } & Identificação do estudo \\
\hline $\begin{array}{l}\text { Hijama ou ventosa terapia com } \\
\text { sangria }\end{array}$ & $\mathrm{A} 1, \mathrm{~A} 8$ \\
\hline Terapia energética & $\mathrm{A} 2$ \\
\hline Prática manipulativa do corpo & $\mathrm{A} 2, \mathrm{~A} 12$ \\
\hline Terapias mente e corpo & $\mathrm{A} 2, \mathrm{~A} 27$ \\
\hline $\begin{array}{l}\text { Produtos naturais/fitoterápicos/plantas } \\
\text { medicinais }\end{array}$ & $\mathrm{A} 2, \mathrm{~A} 4, \mathrm{~A} 5, \mathrm{~A} 7, \mathrm{~A} 9, \mathrm{~A} 10, \mathrm{~A} 12, \mathrm{~A} 13, \mathrm{~A} 14, \mathrm{~A} 15, \mathrm{~A} 16, \mathrm{~A} 17$, \\
Yoga & $\mathrm{A} 18, \mathrm{~A} 19, \mathrm{~A} 20, \mathrm{~A} 21, \mathrm{~A} 23, \mathrm{~A} 25, \mathrm{~A} 10$ \\
\hline Meditação & $\mathrm{A} 10$ \\
\hline Aromaterapia & $\mathrm{A} 10$ \\
\hline Quiropraxia & $\mathrm{A} 6, \mathrm{~A} 10, \mathrm{~A} 32$ \\
\hline Massagem terapêutica & $\mathrm{A} 10$ \\
\hline Naturopatia & $\mathrm{A} 10, \mathrm{~A} 22$ \\
\hline Osteopatia & $\mathrm{A} 10$ \\
\hline Acupuntura & $\mathrm{A} 10, \mathrm{~A} 12$ \\
\hline Mudpack ou banho de lama & $\mathrm{A} 11$ \\
\hline Dietas & $\mathrm{A} 21, \mathrm{~A} 26, \mathrm{~A} 32$ \\
\hline
\end{tabular}




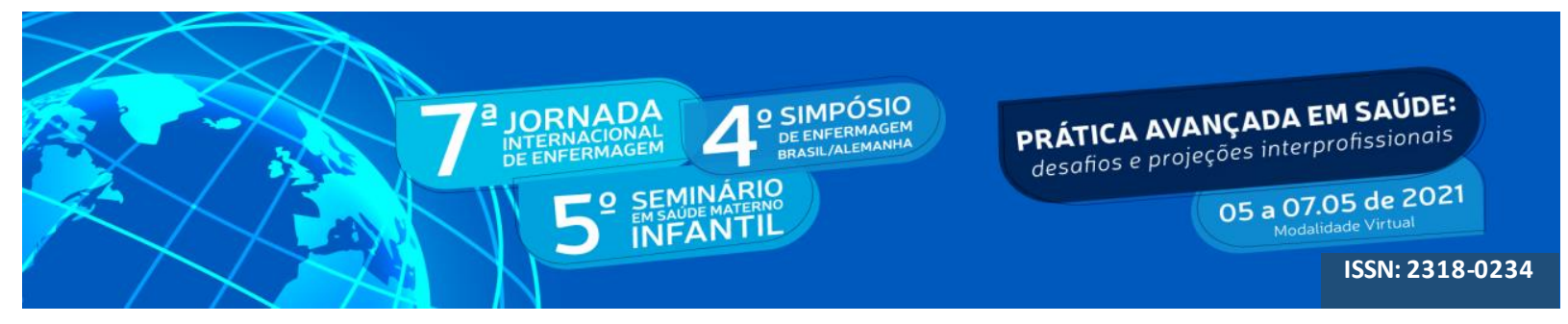

\begin{tabular}{l|c}
\hline Relaxamento guiado por áudio & A24 \\
\hline Homeopatia & A26, A30 \\
\hline Qi terapia & A31 \\
\hline
\end{tabular}

Fonte: Autores.

Dentre os produtos naturais/fitoterápicos ou plantas medicinais que apareceram nos

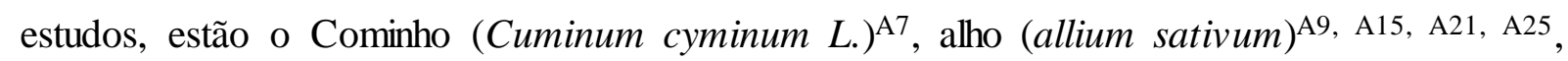
limão ${ }^{\mathrm{A} 9}$, A21, A25, ervas chinesas (Tian-Ma-Gou-Teng-Yin, Gout-Teng-San e Liu-Wei-DiHuang-Wang) ${ }^{\mathrm{A} 12}$, Virechana (composto de ervas para purgação terapêutica) ${ }^{\mathrm{A} 17}$, Basti

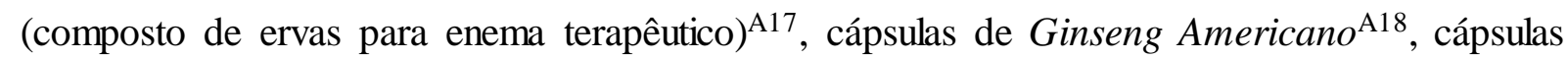
de Salvia officinalis ${ }^{\mathrm{A} 19}$, cápsulas de Pueraria mirifica ${ }^{\mathrm{A} 19}$, suco de romã ${ }^{\mathrm{A} 20}$, visco ou erva-depassarinho $^{\mathrm{A} 21}$ Lavandula stoechas ou rosmaninho ${ }^{\mathrm{A} 21}$, fitoterápico Hempedu Bumi Plus ${ }^{\mathrm{A} 28}$, folhas de mamão ${ }^{\mathrm{A} 28}$ e composto de ervas do Caribe $(b u s h)^{\mathrm{A} 29}$.

No que se refere a efetividade das MTCI testadas nos ECR, a ventosa terapia ou Hijama, mostrou resultados positivos em dois estudos, sendo que um deles mostrou redução da pressão arterial sistólica (PAS) ao longo de três sessões ${ }^{\mathrm{A} 1}$, já no outro, houve redução imediata da PAS, e após quatro semanas de acompanhamento, a pressão arterial média no grupo de intervenção foi $8,4 \mathrm{mmHg}$ menor do que no grupo controle (realizou-se três sessões a cada dois dias). Após oito semanas, não houve diferenças significativas nas pressões arteriais entre os grupos intervenção e controle ${ }^{\mathrm{A} 8}$.

O ECR utilizando o fitoterápico de cápsulas de Ginseng Americano 3g, reduziram significativamente a PAS em 11,7\% (P<0,001) em 12 semanas $^{\mathrm{A} 18}$. Já um ECR mostrou que o consumo de $150 \mathrm{ml} /$ dia de suco de Romã por duas semanas tem efeito hipotensor ${ }^{\mathrm{A} 20}$. Outra terapia testada foi o relaxamento guiado por áudio, que obteve resultados positivos após 12 sessões, três vezes por semana, com redução significativa da PAS e pressão arterial diastólica (PAD) nos idosos participantes ${ }^{\mathrm{A} 24}$. ECR testou a $Q i$ terapia, em que os participantes receberam 10 minutos de aplicação, deitados, e houve diminuição significativa da PAS e $\mathrm{PAD}^{\mathrm{A} 31}$. A terapia $Q i$ ou Qigong mostrou efeitos promissores em estudo, mas que precisam ser melhor consolidados (WONG et al, 2018).

\section{CONCLUSÃO}




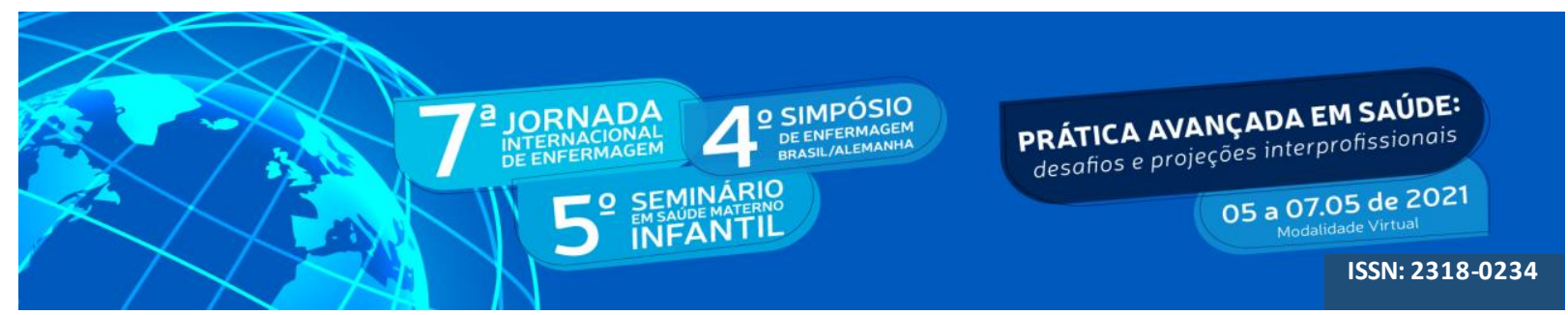

O estudo mostrou uma prevalência de utilização de produtos naturais/fitoterápicos ou plantas medicinais no controle da HAS, sendo que as cápsulas de Ginseng Americano 3g e o consumo de $150 \mathrm{ml} /$ dia de suco de Romã mostraram evidências significativas em ECR realizados. Ademais, a ventosa terapia, relaxamento guiado por áudio e a $Q i$ terapia também trouxeram evidências relevantes.

\section{REFERÊNCIAS}

MELNYK, B. M.; FINEOUT-OVERHOLT, E. Evidence-based practice in nursing \& healthcare: a guide to best practice. $2^{\mathrm{a}}$ ed. Philadelphia: Lippincot Williams \& Wilkins, 2011.

MENDES, K. D. S.; SILVEIRA, R. C. C. P.; GALVÃO, C. M. Revisão integrativa: método de pesquisa para a incorporação de evidências na saúde e na enfermagem. Texto Contexto Enferm, v. 17, n. 4, p. 758-64, 2008.

MOHER, D. et al. The PRISMA Group (2009). Preferred Reporting Items for Systematic Reviews and Meta-Analyses: The PRISMA Statement. PLoS Med, 2009. NATIONAL CENTER FOR BIOTECHNOLOGY INFORMATION. Complementary Therapies. 1986. Disponível em: https://www.ncbi.nlm.nih.gov/mesh/?term=complementary+therapies. Acesso em: 24 Jun. 2019.

VICTOR, R.G. Systemic hypertension: Mechanisms and diagnosis. In: Braunwald's Heart Disease - A Textbook of Cardiovascular Medicine. R.O. Bonow, et al., (Editors), Elsevier Saunders: Philadelphia, 2012.

WONG, A. P. et al. Review: Beyond conventional therapies: Complementary and alternative medicine in the management of hypertension: An evidence-based review. Pak J Pharm Sci. 2018. Disponível em: https://www.researchgate.net/publication/322438249_Beyond_conventional_therapies_Comp lementary_and_alternative_medicine_in_the_management_of_hypertension_An_evidencebased_review

WORLD HEALTH ORGANIZATION. A global brief on Hypertension: Silent killer, global public health crisis. Geneva, 2013a. Disponível em: https://www.ishworld.com/downloads/pdf/global_brief_hypertension.pdf. Acesso em: 03 Jul. 2019.

WORLD HEALTH ORGANIZATION. WHO traditional medicine strategy: 2014-2023. Geneva: World Health Organization; 2013b, 76p. Disponível em: https://www.who.int/medicines/publications/traditional/trm_strategy14_23/en/. 